\title{
Pattern and Predictors of Locoregional Failure in Locally Advanced Breast Cancer Following Neoadjuvant Chemotherapy and Modified Radical Mastectomy with or without Radiotherapy: Retrospective Cohort Analysis of Patients in a Tertiary Hospital Breast Center
}

\author{
Shiela S. Macalindong, ${ }^{1}$ Sigfred B. Lajara, ${ }^{2}$ Jhoanne C. Ynion, ${ }^{3}$ \\ Michele S. Hernandez-Diwa² and Arturo S. Dela Peña ${ }^{1}$ \\ ${ }^{1}$ Department of Surgery, College of Medicine and Philippine General Hospital, University of the Philippines Manila \\ ${ }^{2}$ Department of Pathology, College of Medicine, University of the Philippines Manila \\ ${ }^{3}$ Institute of Clinical Epidemiology, National Institutes of Health, University of the Philippines Manila
}

\begin{abstract}
Objective. The study aimed to identify the pattern and clinicopathologic factors associated with locoregional failure (LRF) in locally-advanced breast cancer (LABC) patients who received neoadjuvant chemotherapy (NAC) and modified radical mastectomy (MRM) with or without adjuvant radiotherapy (RT).
\end{abstract}

Methods. Retrospective cohort analysis of LABC patients who developed LRF following NAC and MRM with or without RT in the Breast Care Center, Philippine General Hospital from 2007-2010 was done. Clinicopathologic and treatment factors were compared between patients who developed and did not develop recurrence using Student's t-tests and Chi-square tests and logistic regression analysis, with $p$ values $\leq 0.05$ considered significant.

Results. A total of 63 patients were included, 34 with locoregional recurrence (LR) and 29 without. Two-year locoregional recurrence rate (LRR) was $54 \%$ with mean time to recurrence at 263 days and chest wall as most common site. Simple logistic regression analysis showed age distribution, pathologic nodal status (pN), percentage positive pathologic lymph nodes, pathologic stage, lymphovascular invasion, and adjuvant RT to be predictors of LR. Furthermore, pN (OR 1.31, Cl 1.07-1.59, $p=0.01$ ) and adjuvant RT (OR 0.14, Cl 0.04-0.53, $p=0.004$ ) were independent predictors of LR on multiple logistic regression analysis. In the subset of patients without adjuvant RT, no independent predictor of LR was found on multiple logistic regression analysis.

Conclusion. Among patients with LABC who received NAC and MRM, locoregional recurrence occurred frequently, usually in the chest wall and within a year of treatment. The absence of adjuvant radiotherapy and increased number of positive pathologic lymph nodes were predictive of locoregional recurrence.

Key Words: locally advanced breast cancer, neoadjuvant chemotherapy, preoperative chemotherapy, recurrence, locoregional failure

Poster presented at the Global Breast Cancer Conference 2015 and $4^{\text {th }}$ International Breast Cancer Symposium, April 23-25, 2015, Jeju Island, South Korea.

Corresponding author: Shiela S. Macalindong, MD

Division of Surgical Oncology,

Head and Neck, Breast, Skin and Soft Tissue, and

Esophagogastric Surgery

Department of Surgery

Philippine General Hospital

University of the Philippines Manila

Taft Avenue, Manila 1000, Philippines

Email: shielamacalindongmd@gmail.com

\section{INTRODUCTION}

Locally-advanced breast cancer (LABC) includes breast cancer "where initial clinical and radiologic evaluation documents advanced disease confined to the breast and regional lymph nodes." ${ }^{\text {N }}$ Neoadjuvant chemotherapy (NAC) is indicated in its initial management to downstage the disease to improve resectability. The risk of locoregional failure (LRF) increases with advancing stage, hence, locoregional recurrences (LR) occur more frequently in 
patients with $\mathrm{LABC} .^{2,3}$ It is important to prevent LR because locoregional control impacts long-term breast cancer mortality. The Early Breast Cancer Trialists' Collaborative Group (EBCTCG) has shown that one breast cancer death over the ensuing 15 years can be avoided for every four LR prevented. ${ }^{4}$ Hence, locoregional control using surgery with or without radiotherapy (RT) is paramount but may be more difficult to achieve in patients with $\mathrm{LABC}$ compared to those with early disease.

Locoregional recurrence rate (LRR) following NAC and mastectomy without radiotherapy in several large, prospective trials range from $5-13 \%$ at 5 to 10 years. ${ }^{5,6}$ Risk factors found to be associated with LR in these studies include clinical tumor and nodal characteristics before NACT, pathological nodal status/breast tumor response after NACT, and breast cancer molecular subtype based on expression of estrogen receptor (ER), progesterone receptor (PR), and human epidermal growth factor receptor 2 neu (Her2/neu). ${ }^{5,6}$ In patients treated with neoadjuvant therapy, risk of recurrence appears to be a function of the interplay of pre-treatment disease characteristics, response to neoadjuvant therapy, and post-treatment disease characteristics. ${ }^{7-11}$

Identification of risk factors for LR after NAC and surgery could help inform decisions regarding optimal application of adjuvant RT to optimize locoregional control. In patients not receiving NAC, adjuvant RT after modified radical mastectomy (MRM) decisions depend on pathologic evaluation of resected specimens and is generally indicated for pathologic tumor size greater than $5 \mathrm{~cm}$, positive pathologic nodes, and/or positive resection margins where re-resection is not feasible., ${ }^{1,12,13}$ Adjuvant RT in this setting reduces risk of LR by about two-thirds. ${ }^{4}$ Following NAC, the indications for adjuvant RT is less clear as NAC alters the resultant pathologic characteristics of tumors and no published randomized trials with impact of adjuvant RT as a predetermined endpoint existed at the time of the study to guide decision. The National Comprehensive Cancer Network (NCCN) currently recommends use of the worst pre-NAC or post-NAC tumor characteristics as basis for adjuvant $\mathrm{RT}$ recommendations. ${ }^{1}$ Hence, LABC, regardless of neoadjuvant treatment response, are generally recommended for adjuvant RT at present. However, use of neoadjuvant therapy has allowed de-escalation of locoregional therapy for breast cancer particularly for early disease, hence the question of whether or not some patients with LABC who received neoadjuvant systemic therapy followed by modified radical mastectomy can be spared of radiotherapy.

The subset of breast cancer patients subjected to NAC locally is far more advanced in stage than the patients included in randomized trials of NAC in breast cancer. There is also very limited literature describing the local experience with NAC for LABC with regard to LR outcomes. Hence, this study aimed to describe the pattern of and the clinicopathologic and treatment variables that predict LR to establish baseline information that can be used to evaluate management approaches and outcomes in the light of international standards and inform the development of local guidelines. It also aimed to look specifically at LR outcomes in patients who did not receive adjuvant RT to determine if there is any factor that predicts for low risk of LR as to exclude use of adjuvant RT.

\section{METHODS}

\section{Study Design and Study Population}

This was a retrospective cohort study of patients with or without LR following NAC and MRM with or without adjuvant RT.

Included in the study were patients at least 18 years of age from the Breast Care Center (BCC) of the Philippine General Hospital (PGH) with LABC (American Joint Committee on Cancer [AJCC] $7^{\text {th }}$ edition stage IIIA, IIIB, IIIC) who received NAC followed by MRM from January 1, 2007 to December 31, 2010 with or without adjuvant RT. To be included, patients must have primary unilateral breast cancer, have undergone appropriate metastatic workup according to $\mathrm{BCC}$ protocol (symptom-directed for stage IIIA and routine for stage IIIB-IIIC consisting of chest radiograph, liver ultrasonography and bone scintigraphy), and with at least 2 years follow up from the time of surgery. Patients with current or past history of non-breast malignancy or incomplete medical records were excluded.

Patients were identified through the Surgical Information System (SIS) of the Department of Surgery, Philippine General Hospital and the conference reports of the Surgical Oncology Division. Medical records and surgical pathology materials of eligible patients were reviewed.

\section{Treatment Details}

First-line NAC regimen used in the BCC during the study inclusion period was an anthracycline-based regimen consisting of 5 -fluorouracil $\left(500 \mathrm{mg} / \mathrm{m}^{2}\right)$, adriamycin/ doxorubicin $\left(50 \mathrm{mg} / \mathrm{m}^{2}\right)$, and cyclophosphamide $(500 \mathrm{mg} /$ $\mathrm{m}^{2}$ ) [FAC] given as IV bolus during day 1 and repeated every 21 days for 4 cycles. Clinical tumor response was assessed prior to administration of subsequent cycle of NAC. Clinical tumor response was categorized into complete response (complete disappearance of primary tumor and involved nodes), partial response (at least 50\% decrease in the size of the primary tumor), no response (less than $50 \%$ decrease in size or increase in size by less than $25 \%$ ), or progressive disease (increase in size by $\geq 25 \%$ or appearance of new lesions). If at least a partial response was not achieved after $2^{\text {nd }}$ cycle of NAC or at any time progressive disease was observed, the NAC was shifted to second line regimen which usually consisted of a single agent taxane, docetaxel $\left(60-75 \mathrm{mg} / \mathrm{m}^{2}\right)$, given on day 1 as intravenous drip over 1 hour every 21 days for 4 cycles. If patient cannot be shifted to second-line NAC, outright MRM was done. 
As per BCC protocol, completion of chemotherapy regimen was initiated 4-6 weeks after the surgery. If patients received 4 cycles of neoadjuvant FAC, 2 more cycles of postoperative FAC every 21 days were given. For patients shifted to docetaxel and given 4 cycles preoperatively, 2 more cycles of docetaxel were given post-operatively. Adjuvant RT was advised for all patients following chemotherapy completion.

\section{Statistical Analysis}

Locoregional recurrence (LR) was defined as relapse in the ipsilateral chest wall, axilla, supraclavicular, infraclavicular, or internal mammary areas. Any recurrence outside these sites was considered distant failure. Cytologic or histologic confirmation of the recurrence was not mandatory for study inclusion as long as the recurrence was unequivocally compatible with recurrence as judged by the attending surgeon and had been managed as such. In the absence of histologic or cytologic confirmation of LR, only patients with obvious locoregional recurrence based on clinical and/or radiologic assessment were included. Only LR that occurred as first events or diagnosed simultaneously with distant metastases were included.

Data were collected relating to age, menopausal status, clinical and pathologic stage/tumor size/nodal status/skin involvement, clinical chest wall involvement, NAC regimen and cycles, response rate, NAC completion, shift to second line NAC, estrogen and progesterone receptor status, number of lymph nodes harvested, percentage (\%) positive lymph nodes (obtained by dividing the number of positive lymph nodes by the number of lymph nodes harvested $\mathrm{x}$ 100), histologic grade, lymphovascular invasion, perineural invasion, pathologic margin status, location of closest margin, adjuvant chemotherapy, adjuvant RT, adjuvant hormonal therapy, time to NAC completion (time from first to last NAC for those who completed NAC), time from last NAC to surgery (time from last administration of NAC to MRM for those who completed NAC), time to adjuvant chemotherapy (time from MRM to first cycle of adjuvant chemotherapy), and time to adjuvant RT (time from MRM to first day of adjuvant RT), time to recurrence (time from MRM to first clinical appearance of LR), site of LR, concomitant distant metastases, and locoregional and systemic management of recurrence.

Patients were categorized into those who developed and did not develop LR. The clinicopathologic variables were compared using Student's t-test or Chi-square for continuous or categorical variables, respectively, for these 2 groups. All $p$ values were 2 -sided and $p$ values $\leq 0.05$ were considered significant. Categorical variables that were found to be associated with LR were entered into a simple logistic regression with LR as the dependent variable. All continuous variables were also subjected to simple logistic regression. All simple logistic regression models found to be significantly associated with LR were entered into multiple logistic regression. Stepwise regression was utilized to find the best fitting model. Subset analysis restricting comparison between the two groups to those patients who did not receive adjuvant $\mathrm{RT}$ was done to determine if there was any risk factor associated with low risk of LR as to preclude adjuvant RT. Patients who developed LR who did and did not receive adjuvant $\mathrm{RT}$ were analyzed separately as to time to recurrence, site of LR, and concomitant distant metastases. STATA version 12 statistical software was used.

\section{Ethical Consideration}

This was a non-interventional study. The study was initiated after approval from the University of the Philippines Manila Research Ethics Board. Patients' data were anonymized using identifiers.

\section{RESULTS}

Sixty-three patients were eligible for study inclusion, 34 with LR and 29 without. The median follow-up was 743 days. Two-year locoregional recurrence rate (LRR) was 54\% with average time to recurrence at 263 days. Most recurrences occurred in the chest wall $(94 \%, 32 / 34)$ alone or in combination with other sites, followed by supraclavicular area $(24 \%, 8 / 32)$, axilla $(12 \%, 4 / 34)$, and infraclavicular area $(6 \%, 2 / 34)$. Concomitant metastases at time of locoregional recurrence were common, occurring in 53\% of the 34 patients. Bones and lungs were the most frequent sites of distant failure at $38.9 \%$ each $(7 / 18)$. These were followed by the contralateral breast $(16.7 \%, 3 / 18)$, liver $(11.1 \%, 2 / 18)$, and brain and contralateral axilla (5.6\%, 1/18 each). Majority of patients did not receive any locoregional or systemic management of and following diagnosis of LR, at $64.7 \%(22 / 34)$ and $35.3 \%$ (12/34), respectively. Surgery was the most common locoregional treatment $(23.5 \%, 8 / 34)$ followed by RT $(11.8 \%$, 4/34). Hormonal therapy was the most common systemic treatment received $(29.4 \%, 10 / 34)$ followed by chemotherapy $(26.5 \%, 9 / 34)$ and both chemotherapy and RT $(8.8 \%, 3 / 34)$.

Table 1 provides a comparison of the clinicopathologic and treatment variables in patients who did and did not develop recurrence. Subset of patients who developed recurrence had significantly more subjects who were 50 years or older, more pathologic lymph nodes, higher percentage of pathologically positive lymph nodes, higher pathologic stage, more lymphovascular invasion, fewer neoadjuvant CT cycles, and less subjects who received adjuvant RT.

Table 2 shows the clinicopathologic and treatment variables found to be predictive of recurrence on simple logistic regression analysis. Belonging in age group 3550 and $>50$ years were associated with lower and higher odds of recurrence, respectively, compared to patients $<35$. Higher pathologic lymph node status, higher percentage of pathologic lymph nodes, and presence of lymphovascular invasion significantly increased the risk of recurrence. Pathologic stage was likewise significantly associated with LR. Adjuvant RT decreased risk of recurrence by $93 \%$. 
Table 1. Patient, disease, and treatment characteristics of patients with and without locoregional recurrence in all patients and in patients who did not receive adjuvant radiotherapy

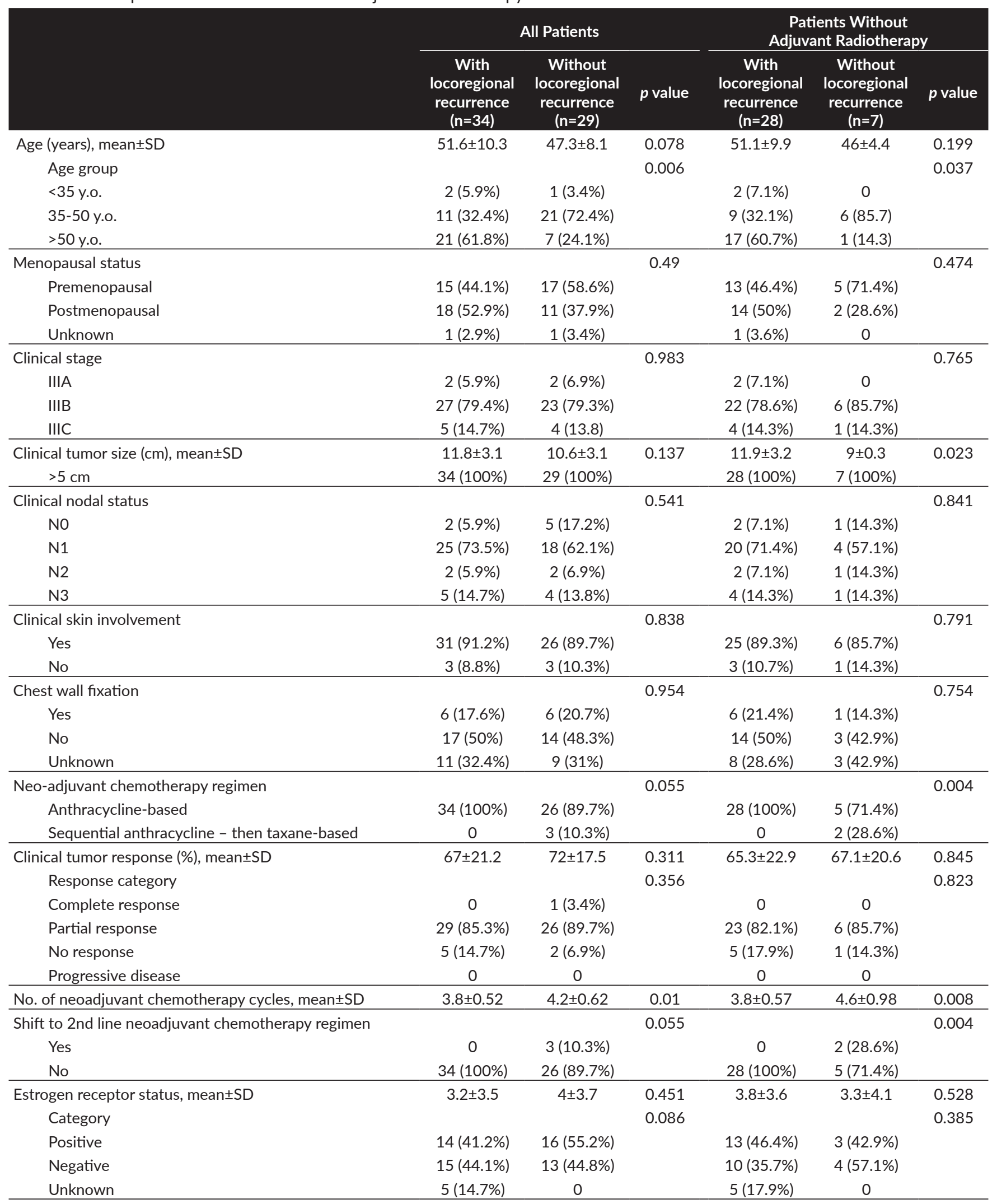


Table 1. continued

\begin{tabular}{|c|c|c|c|c|c|c|}
\hline & \multicolumn{3}{|c|}{ All Patients } & \multicolumn{3}{|c|}{$\begin{array}{c}\text { Patients Without } \\
\text { Adjuvant Radiotherapy }\end{array}$} \\
\hline & $\begin{array}{l}\text { With } \\
\text { locoregional } \\
\text { recurrence } \\
(n=34)\end{array}$ & $\begin{array}{l}\text { Without } \\
\text { locoregional } \\
\text { recurrence } \\
(n=29)\end{array}$ & $p$ value & $\begin{array}{l}\text { With } \\
\text { locoregional } \\
\text { recurrence } \\
(\mathbf{n}=\mathbf{2 8})\end{array}$ & $\begin{array}{l}\text { Without } \\
\text { locoregional } \\
\text { recurrence } \\
(n=7)\end{array}$ & $p$ value \\
\hline Progesterone receptor status, mean $\pm S D$ & $2.2 \pm 2.8$ & $2.9 \pm 3.5$ & 0.388 & $2.4 \pm 3$ & $1.6 \pm 3$ & 0.528 \\
\hline Category & & & 0.095 & & & 0.365 \\
\hline Positive & $12(35.3 \%)$ & $13(44.8 \%)$ & & $10(35.7 \%)$ & $2(28.6 \%)$ & \\
\hline Negative & $17(50 \%)$ & $16(55.2 \%)$ & & $13(46.4 \%)$ & $5(71.4 \%)$ & \\
\hline Unknown & $5(14.7 \%)$ & 0 & & $5(17.9 \%)$ & 0 & \\
\hline Pathologic tumor size $(\mathrm{cm})$, mean $\pm S D$ & $4.3 \pm 3.0$ & $3.8 \pm 3.6$ & 0.516 & $4.5 \pm 3.2$ & $4.45 \pm 3.8$ & 0.967 \\
\hline Category & & & 0.672 & & & 0.98 \\
\hline 0 & 0 & $4(13.8 \%)$ & & 0 & 0 & \\
\hline$\leq 2 \mathrm{~cm}$ & $10(29.4 \%)$ & $7(24.1 \%)$ & & $8(28.6 \%)$ & $2(28.6 \%)$ & \\
\hline $2.1-5 \mathrm{~cm}$ & $14(41.2 \%)$ & $9(31 \%)$ & & $11(39.3 \%)$ & $3(42.9 \%)$ & \\
\hline$>5 \mathrm{~cm}$ & $10(29.4 \%)$ & $9(31 \%)$ & & $9(32.1 \%)$ & $2(28.6 \%)$ & \\
\hline Pathologic lymph node status, mean $\pm S D$ & $8.8 \pm 8.2$ & $1.8 \pm 2.6$ & $<0.00$ & $9.8 \pm 8.7$ & $3 \pm 2.7$ & 0.052 \\
\hline Category & & & $<0.00$ & & & 0.029 \\
\hline 0 & $5(14.7 \%)$ & $13(44.8 \%)$ & & $4(14.2 \%)$ & $1(14.3 \%)$ & \\
\hline $1-3$ & $5(14.7 \%)$ & $12(41.4 \%)$ & & $3(10.7 \%)$ & $4(57.1 \%)$ & \\
\hline $4-9$ & $12(35.3 \%)$ & $3(10.3 \%)$ & & 9 (32.1\%) & $2(28.6 \%)$ & \\
\hline$\geq 10$ & $12(35.3 \%)$ & $1(3.4 \%)$ & & $12(42.9 \%)$ & 0 & \\
\hline No. of lymph nodes harvested, mean \pm SD & $14.9 \pm 6.6$ & $13.7 \pm 4.6$ & 0.415 & $15.1 \pm 7.2$ & $13 \pm 4.3$ & 0.458 \\
\hline Category & & & 0.81 & & & 0.791 \\
\hline$<10$ & $4(11.8 \%)$ & $4(13.8 \%)$ & & $3(10.7 \%)$ & $1(14.3 \%)$ & \\
\hline$\geq 10$ & $30(88.2 \%)$ & $25(86.2 \%)$ & & $25(89.3 \%)$ & $6(85.7 \%)$ & \\
\hline$\%$ positive lymph nodes, mean \pm SD & $55.7 \pm 34.5$ & $14 \pm 20$ & $<0.00$ & $60.2 \pm 34.4$ & $26.2 \pm 23.8$ & 0.02 \\
\hline Category & & & $<0.00$ & & & 0.091 \\
\hline$<20 \%$ & $7(21.2 \%)$ & $22(75.9 \%)$ & & $4(14.8 \%)$ & $3(42.9 \%)$ & \\
\hline$\geq 20 \%$ & $26(78 . \%)$ & $7(24.1 \%)$ & & $23(85.2 \%)$ & $4(57 \%)$ & \\
\hline Pathologic staging & & & 0.009 & & & 0.326 \\
\hline Pathologic complete response & 0 & $3(10.3 \%)$ & & 0 & 0 & \\
\hline 1 & $2(5.9 \%)$ & $3(10.3 \%)$ & & $2(7.1 \%)$ & $1(14.3 \%)$ & \\
\hline IIA & $5(14.7 \%)$ & $6(20.7 \%)$ & & $3(10.7 \%)$ & $1(14.3 \%)$ & \\
\hline IIB & $2(5.9 \%)$ & $7(24.1 \%)$ & & $1(3.6 \%)$ & $1(14.3 \%)$ & \\
\hline IIIA & $9(26.5 \%)$ & $4(13.8 \%)$ & & $7(25 \%)$ & $2(28.6 \%)$ & \\
\hline IIIB & $4(11.8 \%)$ & $5(17.2 \%)$ & & $3(10.7 \%)$ & $2(28.6 \%)$ & \\
\hline IIIC & $12(35.3 \%)$ & $1(3.4 \%)$ & & $12(42.9 \%)$ & 0 & \\
\hline Histologic grade & & & 0.089 & & & 0.102 \\
\hline 1 & 0 & $1(3.4 \%)$ & & 0 & 0 & \\
\hline 2 & $12(35.3 \%)$ & $16(55.2 \%)$ & & $10(35.7 \%)$ & $5(71.4 \%)$ & \\
\hline 3 & $20(58.8 \%)$ & 9 (31\%) & & $17(60.7 \%)$ & $2(28.6 \%)$ & \\
\hline Unknown & $2(5.9 \%)$ & $3(10.3 \%)$ & & $1(3.6 \%)$ & 0 & \\
\hline Lymphovascular invasion & & & 0.008 & & & 0.546 \\
\hline Yes & $30(88.2 \%)$ & $17(58.6 \%)$ & & $26(92.9 \%)$ & $6(85.7 \%)$ & \\
\hline No & $3(8.8 \%)$ & $12(41.4 \%)$ & & $2(7.1 \%)$ & $1(14.3 \%)$ & \\
\hline Unknown & $1(2.9 \%)$ & 0 & & 0 & 0 & \\
\hline Perineural invasion & & & 0.157 & & & 0.35 \\
\hline Yes & $9(26.5 \%)$ & $3(10.3 \%)$ & & 9 (32.1\%) & $1(14.3 \%)$ & \\
\hline No & $24(70.6 \%)$ & 26 (89.7\%) & & $19(67.9 \%)$ & $6(85.7 \%)$ & \\
\hline Unknown & $1(2.9 \%)$ & 0 & & 0 & 0 & \\
\hline
\end{tabular}


Table 1. continued

\begin{tabular}{|c|c|c|c|c|c|c|}
\hline & \multicolumn{3}{|c|}{ All Patients } & \multicolumn{3}{|c|}{$\begin{array}{l}\text { Patients Without } \\
\text { Adjuvant Radiotherapy }\end{array}$} \\
\hline & $\begin{array}{l}\text { With } \\
\text { locoregional } \\
\text { recurrence } \\
(n=34)\end{array}$ & $\begin{array}{l}\text { Without } \\
\text { locoregional } \\
\text { recurrence } \\
(n=29)\end{array}$ & $p$ value & $\begin{array}{l}\text { With } \\
\text { locoregional } \\
\text { recurrence } \\
(n=28)\end{array}$ & $\begin{array}{l}\text { Without } \\
\text { locoregional } \\
\text { recurrence } \\
(n=7)\end{array}$ & $p$ value \\
\hline Skin involvement & & & 0.38 & & & 1 \\
\hline Yes & $9(26.5 \%)$ & $5(17.2 \%)$ & & $8(28.6 \%)$ & $2(28.6 \%)$ & \\
\hline No & $25(73.5 \%)$ & $24(82 \%)$ & & $20(71.4 \%)$ & $5(71.4 \%)$ & \\
\hline Margin status $(\mathrm{cm})$, mean $\pm S D$ & $1.04 \pm 0.81$ & $0.83 \pm 0.57$ & 0.251 & $0.93 \pm .75$ & $0.64 \pm 0.39$ & 0.334 \\
\hline Category & & & 0.578 & & & 0.664 \\
\hline Positive & $1(2.9 \%)$ & 0 & & $1(3.6 \%)$ & 0 & \\
\hline Negative & $31(91.2 \%)$ & $28(96.6 \%)$ & & $25(89.3 \%)$ & $7(100 \%)$ & \\
\hline Close & $2(5.9 \%)$ & $1(3.4 \%)$ & & $2(7.1 \%)$ & 0 & \\
\hline Closest margin & & & 0.467 & & & 0.612 \\
\hline Basal & $33(97.1 \%)$ & 25 (100\%) & & $27(96.4 \%)$ & $7(100 \%)$ & \\
\hline Superior & $1(2.9 \%)$ & 0 & & 0 & 0 & \\
\hline Inferior & $1(2.9 \%)$ & 0 & & 0 & 0 & \\
\hline Unknown & $1(2.9 \%)$ & 0 & & $1(3.6 \%)$ & 0 & \\
\hline $\begin{array}{l}\text { Time to neoadjuvant chemotherapy completion (days), } \\
\text { mean } \pm \text { SD }\end{array}$ & $88.5 \pm 41.8$ & $88.4 \pm 35.1$ & 0.990 & $93.8 \pm 45.6$ & $110.6 \pm 57.3$ & 0.429 \\
\hline $\begin{array}{l}\text { Time from last neoadjuvant chemotherapy to surgery } \\
\text { (days), mean } \pm S D\end{array}$ & $46 \pm 24.5$ & $43 \pm 18.1$ & 0.609 & $48.5 \pm 26.6$ & $42.9 \pm 11.9$ & 0.593 \\
\hline Adjuvant chemotherapy & & & 0.122 & & & 0.139 \\
\hline Yes & $27(79.4 \%)$ & 27 (93.1\%) & & $21(75 \%)$ & $7(100 \%)$ & \\
\hline No & $7(20.6 \%)$ & $2(6.9 \%)$ & & $7(25 \%)$ & 0 & \\
\hline Time to adjuvant chemotherapy (days), mean \pm SD & $44.4 \pm 23.8$ & $49.9 \pm 31.3$ & 0.489 & $46.8 \pm 27$ & $54.7 \pm 20.2$ & 0.49 \\
\hline Adjuvant hormonal therapy & & & 0.179 & & & 0.863 \\
\hline Yes & $13(38.2 \%)$ & $16(55.2 \%)$ & & $11(39.3 \%)$ & $3(42.9 \%)$ & \\
\hline No & $21(61.8 \%)$ & $13(44.8 \%)$ & & $17(60.7 \%)$ & $4(57.1 \%)$ & \\
\hline Adjuvant radiotherapy & & & $<0.00$ & - & - & \\
\hline Yes & $6(17.6 \%)$ & $22(75.9 \%)$ & & & & \\
\hline No & $28(82.4 \%)$ & $7(24.1 \%)$ & & & & \\
\hline Time to adjuvant radiotherapy (days), mean \pm SD & $115.4 \pm 50.9$ & $187.1 \pm 164.2$ & 0.352 & & & \\
\hline
\end{tabular}

Table 3 shows the independent risk factors for LR based on multiple logistic regression analysis using the stepwise regression method to find the best fitting model. Only pathologic lymph node status and adjuvant RT were found to be independent predictors of LR. Having pathologically involved lymph nodes increased recurrence risk by $31 \%$ while adjuvant RT decreased the risk by $86 \%$.

Among patients who developed LR, 82.4\% (28/34) did not have adjuvant RT. Time to recurrence was not significantly different between those who received and did not receive adjuvant RT (357 versus 243 days, $p=0.154$ ) but was shorter for the latter. Pattern of locoregional recurrence was the same for those who did and did not receive adjuvant RT, with the chest wall followed by the supraclavicular area being the most common sites.

Additionally, $78.6 \%$ of patients who received adjuvant RT did not develop LR during the 2-year follow-up while $21.4 \%$ developed recurrence.
Comparison of clinicopathologic and treatment factors between those who did and did not develop recurrence among the subset of patients who did not receive adjuvant RT are presented in Table 1 . Among patients who did not receive adjuvant $\mathrm{RT}$, majority $(80 \%, 28 / 35)$ developed LR within 2 years of surgery. In this restricted subset, age group, clinical tumor size, number of neoadjuvant CT cycles, shift to second-line neoadjuvant $\mathrm{CT}$, neoadjuvant $\mathrm{CT}$ regimen, pathologic lymph node status category, and percentage of positive lymph nodes were significantly different between those who developed and did not develop LR. More patients aged greater than 50 years developed recurrence. Patients who developed recurrence had significantly more pathologic lymph nodes, higher percentage positive lymph nodes, larger average clinical tumor size, and fewer NAC cycles received. The group that had recurrence did not include any patients who were shifted to second line NAC nor received sequential anthracycline- and taxane-based NAC. 
Table 2. Simple logistic regression analysis of factors associated with locoregional recurrence

\begin{tabular}{|c|c|c|c|c|c|}
\hline & Odds Ratio & Standard Error & $p$-value & $\begin{array}{l}\text { 95\% Confidence } \\
\text { Interval }\end{array}$ & $p$-value of model \\
\hline \multicolumn{6}{|l|}{ Age Group (years) } \\
\hline$<35$ (Constant) & 2.00 & 2.45 & 0.57 & $0.18-22.06$ & 0.01 \\
\hline $35-50$ & 0.26 & 0.34 & 0.30 & $0.02-3.22$ & \\
\hline$>50$ & 1.50 & 1.95 & 0.76 & $0.12-19.18$ & \\
\hline \multicolumn{6}{|l|}{ Pathologic Lymph Node Status } \\
\hline Negative (Constant) & 0.30 & 0.13 & 0.01 & $0.13-0.70$ & 0.00 \\
\hline Positive & 1.40 & 0.13 & 0.00 & $1.16-1.69$ & \\
\hline \multicolumn{6}{|l|}{ Pathologic Lymph Node Grouping } \\
\hline 0 (Constant) & 0.38 & 0.20 & 0.07 & $0.14-1.08$ & 0.00 \\
\hline $1-3$ & 1.08 & 0.81 & 0.92 & $0.25-4.70$ & \\
\hline $4-9$ & 10.40 & 8.66 & 0.01 & $2.03-53.20$ & \\
\hline$\geq 10$ & 31.20 & 36.39 & 0.00 & $3.17-306.83$ & \\
\hline \multicolumn{6}{|l|}{ \% Positive Lymph Nodes } \\
\hline 0 (Constant) & 0.25 & 0.11 & 0.00 & $0.11-0.61$ & 0.00 \\
\hline \% Positive Lymph Nodes & 119.82 & 145.47 & 0.00 & 11.09-1294.09 & \\
\hline \multicolumn{6}{|l|}{ \% Positive Lymph Nodes } \\
\hline$<20 \%$ (Constant) & 0.32 & 0.14 & 0.01 & $0.14-0.74$ & 0.00 \\
\hline$\geq 20 \%$ & 12.12 & 7.36 & 0.00 & $3.69-39.82$ & \\
\hline \multicolumn{6}{|l|}{ Pathologic Staging } \\
\hline pCR (Constant) & 12.00 & 12.49 & 0.02 & $1.56-92.29$ & 0.01 \\
\hline IA & 0.06 & 0.08 & 0.04 & $0.00-0.84$ & \\
\hline IIA & 0.07 & 0.08 & 0.03 & $0.01-0.74$ & \\
\hline IIB & 0.02 & 0.03 & 0.00 & $0.00-0.31$ & \\
\hline IIIA & 0.19 & 0.23 & 0.16 & $0.02-1.98$ & \\
\hline IIIB & 0.07 & 0.08 & 0.03 & $0.01-0.75$ & \\
\hline $\mathrm{IIIC}$ & - & & & & \\
\hline \multicolumn{6}{|l|}{ Lymphovascular Invasion } \\
\hline No (Constant) & 0.25 & 0.16 & 0.03 & $0.07-0.89$ & 0.00 \\
\hline Yes & 7.06 & 5.04 & 0.01 & $1.74-28.57$ & \\
\hline \multicolumn{6}{|l|}{ Adjuvant Radiotherapy } \\
\hline No (Constant) & 4.00 & 1.69 & 0.00 & $1.75-9.16$ & 0.00 \\
\hline Yes & 0.07 & 0.04 & 0.00 & $0.02-0.23$ & \\
\hline
\end{tabular}

Table 3. Multiple logistic regression analysis of significant factors associated with locoregional recurrence (best fitting model)

\begin{tabular}{lcccc} 
& Odds Ratio & Standard Error & $p$ value & 95\% Confidence Interval \\
Pathologic lymph node status & 1.31 & 0.13 & 0.01 & $1.07-1.59$ \\
Adjuvant radiotherapy & 0.14 & 0.10 & 0.004 & $0.04-0.53$ \\
\hline Pvalue & & &
\end{tabular}

$p$ value of model: $p<0.00$

goodness-of-fit test: $p=0.797$

Results of simple logistic regression analysis of clinicopathologic variables restricted to patients who did not receive adjuvant RT to identify independent predictors of locoregional recurrence risk in the absence of adjuvant RT are shown in Table 4. Age group 35-50, clinical tumor size, pathologic lymph node status, and percentage positive pathologic lymph nodes were found to be significant predictors of recurrence. Age group 3550 was associated with decreased risk of recurrence while the other 3 factors were associated with increased risk.
Multiple logistic regression and stepwise regression failed to show any significant independent predictor of recurrence in this subset of patients.

\section{DISCUSSION}

In this study, LABC treated with NAC and MRM was associated with a very high LR. Residual positive pathologic nodes following NAC and absence of adjuvant RT were associated with increased risk of recurrence. 
Table 4. Simple logistic regression analysis of factors associated with locoregional recurrence in patients who did not receive adjuvant radiotherapy

\begin{tabular}{|c|c|c|c|c|c|}
\hline & Odds Ratio & Standard Error & $p$-value & $\begin{array}{l}\text { 95\% Confidence } \\
\text { Interval }\end{array}$ & $\begin{array}{l}p \text {-value of } \\
\text { the model }\end{array}$ \\
\hline \multicolumn{6}{|l|}{ Age Group (years) } \\
\hline Constant & 17 & 17.49 & 0.006 & $2.26-127.74$ & 0.013 \\
\hline $35-50$ & 0.09 & 0.10 & 0.04 & $0.01-0.85$ & \\
\hline$>50$ & - & & & & \\
\hline \multicolumn{6}{|l|}{ Clinical Tumor Size } \\
\hline Constant & 0.00 & 0.01 & 0.10 & $0.00-2.63$ & 0.01 \\
\hline Clinical Tumor Size & 1.99 & 0.70 & 0.05 & $1.00-3.98$ & \\
\hline \multicolumn{6}{|l|}{ Pathologic Lymph Node Status } \\
\hline Constant & 1.08 & 0.71 & 0.91 & $0.30-3.93$ & 0.01 \\
\hline Pathologic Lymph Node Status & 1.28 & 0.16 & 0.06 & $0.99-1.64$ & \\
\hline \multicolumn{6}{|l|}{ \% Positive Lymph Nodes } \\
\hline Constant & 0.93 & 0.65 & 0.91 & $0.23-3.66$ & 0.02 \\
\hline \% Positive Lymph Nodes & 29.66 & 48.01 & 0.04 & $1.24-707.80$ & \\
\hline
\end{tabular}

The LRR reported in this study is much higher than the 5-20\% 5-10 year rates reported in literature for patients treated with NAC followed by surgery $5,6,8,10,11,14,15$ This may be due to inclusion of very advanced stage of breast cancer including inflammatory breast cancer, the use of predominantly anthracycline based-NAC, limited application of adjuvant RT, non-use of targeted therapy like herceptin or pertuzumab where it may have been indicated, and inclusion of LR cases occurring concurrently with distant metastases in this study.

Systemic treatment for breast cancer affects both survival and recurrence outcomes. The high LRR in this study may be due to the choice of the neoadjuvant chemotherapy regimen. At the time of the treatment of the patients included in the study, CAF regimen given in a "sandwich" method, that is 4 cycles before and 2 cycles after surgery, was standard. From the results of the National Surgical Adjuvant Breast and Bowel Project (NSABP) 18 and 27 trials, addition of taxane to an anthracycline regimen and completion of the entire chemotherapy regimen preoperatively increased pathologic complete response rates ( $\mathrm{pCR}$ ), that is, the absence of invasive disease in the breast and lymph nodes. ${ }^{16} \mathrm{pCR}$ is associated with better survival outcome. ${ }^{16}$ From the same studies, addition of docetaxel in the NAC regimen decreased 10-year cumulative incidence of LR by 8.5\%. ${ }^{6}$ Hence, for LABC, more appropriate NAC regimen should include both an anthracycline and taxane. Where an anthyracycline-based regimen remains the only NAC option in settings where access to taxane is difficult, giving the full regimen preoperatively may be preferable.

The general recurrence pattern in the study is similar with foreign reports showing the chest wall followed by the supraclavicular area as most common LR sites for patients treated and not treated with NAC. $2,8,10-11$

More than half of the patients with LR had concomitant distant metastases at diagnosis. This may be an underestimation given the high percentage of patients who were lost to follow-up after initial LR diagnosis and were, consequently, inadequately investigated for metastases. The low rate of isolated LR may partially account for the limited number of patients who received locoregional treatment for the recurrence. The Philippine College of Surgeons (PCS) guidelines recommend restaging following diagnosis of recurrence. ${ }^{17}$ The results of the study support this recommendation and validate the practice of routine metastatic workup for patients with recurrence in the BCC. Detection of concurrent distant metastasis will shift treatment decisions in favor of systemic rather than locoregional treatment.

Only $56 \%$ of patients in the study had cytologic or histologic confirmation of the recurrence, a significant limitation of the study. This is inconsistent with PCS and NCCN guidelines that recommend pathologic confirmation of recurrence whenever feasible. ${ }^{1,17}$ The low biopsy rate may be due to the high loss to follow-up rate within a month of clinical detection of recurrence at 32\% and the concomitant diagnosis of distant metastases at the time of locoregional recurrence in about half of the patients. The latter does not negate the importance of securing biopsy of the locoregional recurrence, as apart from providing pathologic confirmation, it is important for retesting for hormone receptor status particularly if it was initially unknown or negative. Hormone receptor status may be discordant between the primary tumor and the recurrence, hence, influencing selection of systemic treatment. ${ }^{1}$ In the study, only patients with unequivocal clinical or radiologic findings of locoregional recurrence were included.

The clinicopathologic and treatment factors that were found to be predictive of locoregional recurrence risk in this study: age, pathologic lymph node status, pathologic stage, lymphovascular invasion, and receipt of adjuvant RT, have been previously identified in several foreign reports that 
included both neoadjuvant and adjuvant chemotherapy to be associated with recurrence risks., ${ }^{2,9-11,18}$ The higher risk of recurrence in older patients in this study is in contrast with foreign report showing greater risk for younger, premenopausal patients and may be due to different age stratification and the non-association of menopausal status and recurrence risk in this study. ${ }^{2}$

Similar to foreign reports, a higher number of residual pathologic lymph nodes particularly 4 or more independently increases the risk of LR. ${ }^{6,19}$ In the study, patients who developed LR had a mean of 9 positive lymph nodes whereas those who did not develop LR had a mean of only 2 positive nodes. Apart from absolute number of pathologic lymph nodes, percentage of positive pathologic lymph nodes was included in the analysis due to concern for decreased axillary nodal harvest following NAC. ${ }^{20}$ Having at least $20 \%$ positive lymph nodes was reported to significantly increase risk of recurrence, similar to the study results. ${ }^{10}$

Achievement of $\mathrm{pCR}$ was found to correlate with lower recurrence risk in foreign reports but was not observed in this study, although none of the patients with $\mathrm{pCR}$ developed LR. ${ }^{6,21}$ This could be due to the very low $\mathrm{pCR}$ rate $(4.8 \%)$ seen in the study even compared to NAC trials that used anthyracyline-based regimen alone (13\%). ${ }^{16}$ The difficulty in achieving $\mathrm{pCR}$ in this study could be due to the large size of tumors and extent of nodal involvement before NAC.

Interestingly, other variables that correlate with LR risk in foreign reports were not found to be significant in this study. High clinical/pre-NAC stage, particularly stage III disease, was significantly associated with LR on multivariate analysis in some reports. ${ }^{6,10}$ ER-negativity was often associated with high risk of LRF but not found in this study to be significant. ${ }^{10,19}$

The results of this study suggest that adjuvant RT following NAC and MRM decreases the risk of LR by as high as $86 \%$. This is consistent with a large retrospective study of patients treated with NAC and mastectomy (with or without RT) in the MD Anderson Cancer Center which showed that lack of radiation treatment was the greatest hazard associated with LR (HR 4.7, $\mathrm{p}<0.001$ ) on multivariate analysis. ${ }^{10}$ Radiation significantly reduced the risk in patients with clinical T3 or T4 tumors, clinical $\mathrm{N} 2$ or N3 disease, clinical IIB or greater disease, at least 4 pathologic lymph nodes, or pathologic tumor size greater than $2 \mathrm{~cm} .{ }^{10}$ Radiation improved LRR even in clinical stage III patients who achieved pCR. ${ }^{10}$

Majority of patients who developed a recurrence in the study did not receive adjuvant RT. Moreover, adjuvant RT appears to delay recurrence in cases where it occurred although the time difference was not statistically significant. The findings in the study appear to validate the existing practice of doing RT to all patients with $\mathrm{LABC}$ who underwent NAC followed by surgery regardless of the response to NAC and resultant pathologic staging. No clinicopathologic variable was found strong enough to predict low recurrence risk in the absence of adjuvant RT as to preclude its use. In contrast, a foreign retrospective review of 134 patients with stage II and III breast cancer treated with NAC and mastectomy showed that omission of adjuvant RT in patients who achieved pathologically negative lymph nodes did not increase LR. ${ }^{9}$ However, this should be interpreted with caution given the retrospective nature of the study, the limited number of patients, and inclusion of earlier stage disease.

Another retrospective review looked into pathologic factors predictive of recurrence in patients who had mastectomy without RT who received either neoadjuvant or adjuvant chemotherapy. ${ }^{7}$ Despite higher initial clinical stage in the NAC group, pathologic tumor size and number of lymph nodes were less compared to the adjuvant group. Nonetheless, the LR rate was still higher in the NAC group. This suggests that NAC response and resultant pathologic tumor characteristics cannot reliably select for patients who would not benefit from the addition of adjuvant RT in those for whom it is indicated based on pre-neoadjuvant disease characteristics such as those with LABC.

While use of NAC may lead to de-escalation of locoregional therapy in some clinical scenarios particularly in early breast cancer, findings from this study suggest that for LABC, adjuvant radiotherapy remains important to decrease LR risk.

Despite negative margins in $94 \%$ of patients, a high LRR was still observed in the study. This underscores that recurrence is not a function of margin alone but of the disease itself, hence the recommendation for adjuvant RT for tumors $>5 \mathrm{~cm}$ or $\geq 4$ pathologic lymph nodes. ${ }^{1,12}$ Negative margins, however, remains important for locoregional control and adjuvant RT is not intended to substitute for inadequate surgery. In the study, 3 of the 4 patients with positive or close margins, none of whom received adjuvant RT, subsequently developed LR. The other patient who did not develop recurrence received adjuvant RT. The nonassociation of margin status with LR in the study may be due to limited number of observations with positive or close margins. The basal margin was reported to be the closest margin in almost all cases. Hence, en bloc resection of part of or the entire pectoralis major muscle where indicated must be considered to achieve negative margins.

The high recurrence rate in the chest wall observed despite negative margins raises concerns about skin margin adequacy. The current institutional practice in doing MRM following NAC is to resect the involved skin around the tumor noted at the time of surgery with a $2 \mathrm{~cm}$ gross surgical margin and not based on pre-NAC skin involvement. While none of the pathologic reports showed close involvement of skin margins, reports have shown that detection of microfocus of tumor following NAC may be difficult to detect with traditional pathologic sectioning techniques. ${ }^{22}$ 
NAC does not always cause uniform tumor regression and multifocal residual tumor due to non-uniform destruction can occur. ${ }^{23-24}$ This multifocal residual tumor morphology was most commonly seen in patients with larger tumors and was associated with higher recurrence rates..$^{23-24}$ Resection of involved skin based on pre- or post-NAC skin involvement warrants further investigation, particularly in the setting where adjuvant RT cannot be assured.

In the study, the variables "shift to $2^{\text {nd }}$ line NAC" and NAC regimen "sequential anthracycline- then taxanebased" may be regarded as similar variables since only those who were non-responders to initial FAC regimen were shifted to $2^{\text {nd }}$ line taxane-based therapy. Notably, patients who were non-responders to FAC and not shifted to second line NAC developed recurrence whereas nonresponders who were shifted to taxane-based therapy did not. Moreover, the group of patients that developed recurrence received less number of NAC cycles. This can be attributed to greater number of non-responders to initial FAC regimen in the recurrence group who proceeded directly to MRM instead of being shifted to second-line taxane. Surgery was elected if patients cannot be shifted to $2^{\text {nd }}$ line NAC following non-response to initial regimen to avoid loss of opportunity for locoregional control. However, this course of action appears to result in shortlived locoregional control only. A shift to second line chemotherapy appears to be the more favorable option to achieve locoregional control in patients who failed on initial NAC. Whether the favorable response with shifting to $2^{\text {nd }}$ line regimen is the effect of the shift itself or of the use of taxane is unknown. The NSABP B-27 showed that while addition of docetaxel (T) to preoperative adriamycincyclophosphamide (AC) did not improve disease-free (DFS) and overall survival (OS), it significantly increased the proportion of patients with $\mathrm{pCR}$ compared to preoperative $\mathrm{AC}$ alone. ${ }^{16}$ Patients who achieved $\mathrm{pCR}$ had better DFS and OS than those who did not. ${ }^{16}$

In light of more recent recommendations on neoadjuvant therapy for breast cancer, the studied regimen in the current study and the manner it was given, i.e., with surgery "sandwiched" between cycles, serve as limitations. However, at the time of the study FAC was considered standard protocol in the center. Even at present, the regimen is still widely used in the country particularly where access to more expensive regimens and targeted therapy is limited, hence, the pervasive relevance of the study.

Another study limitation is the lack of significant information on human epidermal growth factor receptor 2 neu (Her2/neu) that precluded adequate assessment of impact of tumor biology as defined by molecular subtype of breast cancer (luminal A, luminal B, Her2-positive or triple negative breast cancer [TNBC]) on LR in these patients. At the time of the study, Her2/neu determination of tumors, although available, was not routinely done in the institution for pragmatic reasons: the additional cost of the test and its utility given the inaccessibility of recommended targeted therapy due to financial limits of most patients. Breast cancer subtype has been found to be a significant predictor of LR with triple negative tumors (ERnegative, PR-negative, and HER2 negative) and Her2positive tumors untreated with trastuzumab having higher risk for LR than luminal A (hormone receptor positive, Her2-negative) tumors. ${ }^{5,21,25}$ Impact of $\mathrm{pCR}$ on LR was found to be subtype-specific in that non-pCR in TNBC breast cancer was particularly associated with LR. ${ }^{21}$ The finding that in Her2-positive breast cancer patients who have not received trastuzumab have higher risk of LR than those who have received it underscores the importance of systemic treatment in decreasing risk of LR. ${ }^{5,25}$

Another study limitation is the small number of patients included due to the follow up requirement of at least 2 years based on the review of 5 NSABP trials which showed a median time to develop isolated locoregional failure at 2 years. ${ }^{2}$ Given that the average time to recurrence observed in this study is less than 1 year, it may be possible to expand the study sample by decreasing the follow-up requirement to 1 year.

This study only looked into LRF as end point. A local study that would look into survival outcomes in this patient population would be of even greater importance to assess impact of treatments for LABC.

Despite its limitations, the study has the advantage of reflecting clinical practice in LABC management locally particularly given the limitation in administration of ideal systemic treatments including addition of taxane and Her2-targeted therapy, where indicated.

\section{CONCLUSIONS}

More than half of patients who initially received neoadjuvant CT followed by MRM with or without adjuvant RT had LR within 2 years of follow-up. Majority of the recurrences occurred in the chest wall and more than half had associated distant metastases. Presence of pathologic lymph nodes was found to be an independent predictor of increased risk of LR while receipt of adjuvant RT was found to independently predict for lower risk. No clinicopathologic variable was found to significantly predict for low risk of recurrence in the absence of adjuvant RT.

\section{Statement of Authorship}

All authors participated in data collection and analysis, and approved the final version submitted.

\section{Author Disclosure}

All authors declared no conflicts of interest.

\section{Funding Source}

This paper was funded by the principal investigator. 


\section{REFERENCES}

1. National Comprehensive Cancer Center Network [Internet]. NCCN Clinical Practice Guidelines in Oncology. Breast Cancer Guidelines version 3.2014. [cited 2019 Jul]. Available from https://www.nccn.org/ professionals/physicians_gls/pdf/breast.pdf

2. Taghian A, Jeong JH, Mamounas E, Anderson S, Bryant J, Deutsch M, et al. Patterns of locoregional failure in patients with operable breast cancer treated by mastectomy and adjuvant chemotherapy with or without tamoxifen and without radiotherapy: Results from five National Surgical Adjuvant Breast and Bowel Project randomized trials. J Clin Oncol. 2004; 22(21):4247-54.

3. Wallgren A, Bonetti M, Gelber RD, et al. Risk factors for locoregional recurrence among breast cancer patients: Results from International Breast Cancer Study Group Trials I through VII. J Clin Oncol. 2003; 21(7):1205-13.

4. Early Breast Cancer Trialists' Collaborative Group (EBCTCG). Effects of radiotherapy and of differences in the extent of surgery for early breast cancer on local recurrence and 15-year survival: an overview of the randomized trials. Lancet. 2005; 366(9503):2087-106.

5. Gillon P, Touati N, Breton-Callu C, Slaets L, Cameron D, Bonnefoi $\mathrm{H}$. Factors predictive of locoregional recurrence following neoadjuvant chemotherapy in patients with large operable or locally advanced breast cancer: An analysis of the EORTC 10994/BIG 1-00 study. Eur J Cancer. 2017; 79:226-234.

6. Mamounas EP, Anderson SJ, Dignam JJ, Bear HD, Julian TB, Geyer $\mathrm{Jr} \mathrm{CE}$, et al. Predictors of locoregional recurrence after neoadjuvant chemotherapy: results from combined analysis of National Surgical Adjuvant Breast and Bowel Project B-18 and B-27. J Clin Oncol. 2012; 30(32);3960-6.

7. Buchholz TA, Katz A, Strom EA, McNeese MD, Perkins GH, Hortobagyi GN, et al. Pathologic tumor size and lymph node status predict for different rates of locoregional recurrence after mastectomy for breast cancer patients treated with neoadjuvant versus adjuvant chemotherapy. Int J Radiat Oncol Biol Phys. 2002; 53(4):880-8.

8. Cance WG, Carey LA, Calvo BF, Sartor C, Sawyer L, Moore DT, et al. Long-term outcome of neoadjuvant therapy for locally advanced breast carcinoma: Effective clinical downstaging allows breast preservation and predicts outstanding local control and survival. Ann Surg. 2002; 236(3):295-303.

9. Le Scodan R, Selz J, Stevens D, Bollet MA, de la Lande B, Daveau $\mathrm{C}$, et al. Radiotherapy for stage II and stage III breast cancer patients with negative lymph nodes after preoperative chemotherapy and mastectomy. Int J Radiat Oncol Biol Phys. 2012; 82(1):e1-7.

10. Huang EH, Tucker SL, Strom EA, McNeese MD, Kuerer HM, Hortobagyi GN, et al. Predictors of locoregional recurrence in patients with locally advanced breast cancer treated with neoadjuvant chemotherapy, mastectomy, and radiotherapy. Int J Radiat Oncol Biol Phys. 2005; 62(2):351-7.

11. Huang EH, Tucker SL, Strom EA, McNeese MD, Kuerer HM, Buzdar AU, et al. Postmastectomy radiation improves local-regional control and survival for selected patients with locally advanced breast cancer treated with neoadjuvant chemotherapy and mastectomy. J Clin Oncol. 2004; 22(23):4691-9.

12. Goldhirsch A, Winer EP, Coates AS, Gelber RD, Piccart-Gebhart $\mathrm{M}$, Thurlimann B, et al. Personalizing the treatment of women with early breast cancer: highlights of the St. Gallen International Expert Consensus on the primary therapy of early breast cancer 2013. Ann Oncol. 2013; 24(9):2206-23.
13. Recht A, Edge SB, Solin LJ, Robinson DS, Estabrook A, Fine RE, et al. Postmastectomy radiotherapy: Clinical practice guidelines of the American Society of Clinical Oncology. J Clin Oncol. 2001; 19(5):1539-69.

14. van der Hage JA, van de Velde CJH, Julien JP, Tubiana-Hulin M, Vandervelden C, Duchateau L. Preoperative chemotherapy in primary operable breast cancer: Results from the European Organization for Research and Treatment of Cancer Trial 10902. J Clin Oncol. 2001; 19(22):4224-37.

15. Brito RA, Valera V, Buzdar AU, Booser DJ, Ames F, Strom E, et al. Long-term results of combined-modality therapy for locally advanced breast cancer with ipsilateral supraclavicular metastases: the University of Texas M.D. Anderson Cancer Center Experience. J Clin Oncol. 2001; 19(3):628-33.

16. Rastogi P, Anderson SJ, Bear HD, Geyer CE, Kahlenberg MS, Robidoux A, et al. Preoperative chemotherapy: updates of National Surgical Adjuvant Breast and Bowel Project Protocols B-18 and B-27. J Clin Oncol. 2008; 26(5):778-85.

17. De Leon-Matsuda M, Laudico AV, Cabaluna ND, et al. Evidencebased clinical practice guidelines on the diagnosis and management of breast cancer part II: locally advanced breast cancer, locally recurrent breast cancer, and metastatic breast cancer. Philippine Journal of Surgical Subspecialties. 2001;56(4):135-164.

18. Taras AR, Thorpe JD, Morris AD, Atwood M, Lowe KA, Beatty JD. Irradiation effect after mastectomy on breast cancer recurrence in patients presenting with locally advanced disease. Am J Surg. 2011; 201(5):605-10.

19. Nakajima N, Oguchi M, Kumai Y, Yoshida M, Inoda H, Yoshioka $\mathrm{Y}$, et al. Clinical outcomes and prognostic factors in patients with stage II-III breast cancer treated with neoadjuvant chemotherapy followed by surgery and postmastectomy radiation therapy in the modern treatment era. Adv Radiat Oncol. 2018; 3(3):271-9.

20. Belanger J, Soucy G, Sideris L, Leblanc G, Drolet P, Mitchell A, et al. Neoadjuvant chemotherapy in invasive breast cancer results in a lower axillary lymph node count. J Am Coll Surg. 2008; 206(4):704-8.

21. Yang J, Morrow M, Modi S, Zhang Z, Krause K, Siu C, et al. The effect of molecular subtype and residual disease on locoregional recurrence in breast cancer patients treated with neoadjuvant chemotherapy and postmastectomy radiation. Ann Surg Oncol. 2015; 22(Suppl 3):S495-501.

22. Wang S, Zhang Y, Yang X, Fan L, Qi X, Chen Q, et al. Shrink pattern of breast cancer after neodjuvant chemotherapy and its correlation with clinical pathological factors. World J Surg Oncol. 2013;11(1):166-71.

23. Chen AM, Meric-Bernstam F, Hunt KK, Thames HD, Oswald MJ, Outlaw ED, et al. Breast conservation after neoadjuvant chemotherapy: the M.D. Anderson Cancer Center Experience. J Clin Oncol. 2004; 22(12):2303-12.

24. Veronesi U, Bonadonna G, Zurrida S, Galimberti V, Greco M, Brambilla $\mathrm{C}$, et al. Conservation surgery after primary chemotherapy in large carcinomas of the breast. Ann Surg.1995; 222(5):612-8.

25. Jwa E, Shin KH, Kim JY, Park YH, Jung S, Lee ES, et al. Locoregional recurrence by tumor biology in breast cancer patients after preoperative chemotherapy and breast conservation treatment. Cancer Res Treat. 2016; 48(4):1363-72. 